\title{
Measuring and modelling arm dynamics to support studies into reducing tremor in individuals with multiple sclerosis
}

\author{
L. P. Ketteringham ${ }^{1}$, S. A. Neild ${ }^{1}$, R. A. Hyde ${ }^{1}$, R. J. S. Jones ${ }^{2}$ \\ \& A. Davies-Smith ${ }^{2}$ \\ ${ }^{1}$ Department of Mechanical Engineering, University of Bristol, UK \\ ${ }^{2} U B H T$, Bristol Royal Infirmary, UK
}

\begin{abstract}
This paper reports on work carried out to date, and future directions for, a project that intends to measure and control tremor in individuals with multiple sclerosis (MS). The project will use computer modelling to simulate the movements involved in upper limb intention tremor, as exhibited by individuals with MS.

The models will be incorporated into a final system that will interpret measurements from sets of microelectromechanical systems (MEMS) sensors positioned over the surfaces of the upper limb to provide position, orientation and movement information. These data will be combined with gross muscle activity data, extracted from surface electromyogram (EMG) measurements, to create a fully dynamic model of the arm movements in real time. This model will be used to develop methods of attenuating the tremor, firstly at the elbow joint, without adversely affecting the underlying intended movement, by providing real-time feedback control of appropriate force input devices. The estimates of movement and the forces involved will also provide clinicians with a useful tool in the quantification of upper limb tremor.

Keywords: dynamic model, upper limb, tremor reduction, multiple sclerosis, MEMS sensors, EMG.
\end{abstract}

\section{Introduction}

MS is the most common disabling neurological condition that affects young adults, the onset of symptoms typically being between 20 and 45 years of age, 
affecting twice as many women as men [1]. Approximately 85,000 people in the UK, 350,000 people in the United States and more than 2.5 million worldwide have MS; it leads to substantial disability in more that $50 \%$ of patients [2].

The symptoms of MS arise as a result of damage to the insulating myelin sheaths of nerve axons, after which the axons can degenerate. MS is classed as an autoimmune disease as it is thought that this demyelination results from attacks by an individual's immune system (likely by myelin-specific CD4 T cells) on their own nervous system [2]. MS is variable in the way in which it can affect individuals, both in the severity of the symptoms and in which systems are affected. This can make diagnosis difficult. New symptoms can develop slowly over time or can take the form of relapse-remission cycles. MS currently is incurable, though several treatments are available which may slow the appearance of new symptoms and reduce the severity of existing symptoms.

Calabresi [1] gives an overview of the symptoms of MS and methods for diagnosing and managing the disease. Among many other symptoms, the muscles can be affected such that they exhibit, stiffness, spasticity, spasms, fatigue and tremor. Especially common in MS is a form of tremor called intention tremor, which exhibits itself not when the person is still, but when they move to carry out an action, such as when reaching or picking up objects. This can be very frustrating, as the further the person reaches, the worse the tremor gets. The uncoordinated movement that results is often referred to as ataxia.

Tremor can affect as many as three quarters of people with MS, though for some it is only slight. $38 \%$ of 100 patients examined by Alusi et al [3] had tremor that was severe enough to be symptomatic. Of those that had tremor in their arms (including non-symptomatic tremor), 36 had bilateral and 9 had unilateral arm tremor, while a further 12 had tremor in one or both arms together with other parts of the body. These proportions may vary in the wider populace, though, as this study was a limited to 100 individuals.

Wrist, elbow and shoulder tremors that occur during intentional movements (listed as postural, kinetic and intention tremors in [3]) are particularly disabling for ADL and were found to have tremor frequency components of between 3 and $7 \mathrm{~Hz}$, with most between 3-5 Hz; the higher frequencies are likely created in the parts of the arm with lower masses, such as the hand and fingers. The distal wrist and elbow tremors were particularly disabling. This tremor is of a lower frequency than 'normal' physiological tremor, which is usually of 8-12 Hz.

Those with severe tremor can find their activities of daily living (ADL) are severely disrupted or impossible to complete without help. This tremor can be exhausting and can also cause acute embarrassment and self-consciousness. Tremor can be somewhat reduced with the aid of physiotherapy and occupational therapy. Maintaining muscle strength and range of movement and learning ways to minimise the effort associated with movements that cause tremor can help to reduce the effects on ADL.

Methods of controlling upper limb tremor will be investigated using a combination of modelling and experimentation. Several candidates for tremor control will be assessed for efficiency and acceptability for tremor damping, first using modelling, then successful candidates will be tested experimentally. These 
will include mechanical methods such as using additional inertia, passive tuned mass dampers, rotary dampers, or active absorbers. Functional electrical stimulation (FES) of the skeletal muscles is another possible candidate for tremor reduction. Modelling this process is likely to be complex, however, and so quantitatively comparing FES to mechanical methods by modelling will be challenging; it may be better to compare experimental FES studies with both modelling and experimental studies on mechanical tremor reduction equipment.

\section{Measuring and controlling tremor}

Controlling tremor in the upper limb is important for maintaining a person's independence, as many of the ADL centre around performing reaching, grasping, and other activities away from the body, where tremor is more likely to occur. This is a non-trivial task, particularly as the movements of the upper limbs are usually more complex and voluntary in nature than those of the lower limbs, due to the nature and greater variety of tasks carried out using the upper limbs.

For any form of tremor control to be used during ADL it should be minimally invasive to these activities. Many previous external orthoses have been conspicuous, cumbersome and difficult to don and doff, reducing uptake in the population they intend to help. Memberg et al [4] report that none of the subjects in their study to restore elbow extension via FES was willing to add an accelerometer to their arm as an input signal to assist with automation of the FES control of their triceps, as they did not want to don an additional device and cable on their arm. Patients preferred a simple, less flexible triceps controller, because those external components were lighter, less visible and less bulky.

Before motion control can be achieved an accurate and reliable system is required for estimating body positions and movements. The route proposed here is to use MEMS sensors, such as miniature accelerometers, gyroscopes (gyros) and magnetometers for measuring both stationary orientation and movement of limb segments, together with EMG sensors for measuring muscle activity. These measurements will be used as inputs to a fully dynamic model of the upper limb that can provide estimates of segment positions, orientations and movements, allowing control of the muscles to reduce tremor.

The MEMS sensor system itself would also provide a useful tool for measuring tremor levels and movement capabilities in individuals during clinical assessment. This would allow assessment of the efficacy of other treatments in a less subjective manner than is currently available. Examples of work using such sensors for measuring body segment positions are can be found in [5] and [6]. These two publications show the progression from using solely accelerometer data to incorporating gyro measurements. Although the system in [5] led to estimates that became unreliable even after a very short time, addition of gyros to the system in [6] gave a far better position and orientation reconstruction estimator over a short test.

Accuracy may be further improved by adding known constraints into the system. The method described by Hyde et al [7], where the body segments are 
treated as a rigid body system joined together at joints with specified degrees of freedom (DOF), will be used to enhance the accuracy of estimates.

Dejnabadi et al [8] used a method where estimates of limb position during gait were reset to the initial position at the end of each gait cycle, eliminating the drift that arises between estimates and reality over time. This approach may be possible for the upper limb, but the time allowed for arm excursions from a default position may have to be longer than a normal gait cycle for the intrusion of regularly returning to a position not to be inconvenient.

\subsection{MEMS measurement system}

MEMS sensors have become widely used in consumer devices and thus much cheaper, especially in the last 2 years. Relatively inexpensive inertial measurement unit (IMU)-type sensors are now commercially available (e.g. the MTx from Xsens Technologies, The Netherlands). Each MTx unit can provide simultaneous readings from three orthogonal axes each of accelerometer, gyro and magnetometer together with computed 3D orientation, while still being housed in a fairly small package size. Positioning several of these over the body's surface can therefore provide orientations of multiple segments simultaneously, allowing analysis of position and movement in multiple DOF.

The addition of magnetometer readings allows a drift-free horizontal angle to be determined, at least when the subject is stationary or moving slowly, which is not possible with accelerometers and gyros alone. Estimation of horizontal angle using accelerometers and gyros would require an initial angle to be known and this to be combined with double-integration of accelerometer and/or integration of gyro readings over time. This method is very susceptible to noise and drift in the readings from these devices.

Inputting readings from the MTx sensors into a dynamic model of the arm should provide estimates of movement and force, allowing control of tremor without adversely affecting underlying intentional movements; these position and movement estimates will also provide an objective clinical assessment tool.

Although the entire arm movement will be estimated from the MTx measurements, work will initially concentrate on controlling the movement only at the elbow, as the rotation of the elbow joint is one of the largest movements in ADL, such as a reaching task, and it is the simplest of the large joints in the upper limb, having only 1 DOF and two actuating muscle groups.

\subsection{EMG and IMU measurement system}

An EMG measurement and recording device was created previously in this project. The intention is to add readings from the MTx devices to the data acquisition (DAQ) program created from the EMG system, so that all readings can be captured simultaneously and used as an input to the dynamic arm model.

A USB-1608FS from Measurement Computing was used for acquiring the EMG data, due to its compact size, light weight, accuracy, speed of acquisition and ability to use on a laptop running from its internal batteries. The laptop was always run from batteries when recording EMG signals to isolate the test subject 
from potentially harmful high voltages and reduce mains noise. The device was housed in a sturdy enclosure that was small, light and portable, so that could be attached to the subject while logging EMG data.

Two Biometrics Ltd. SX230 sensors were attached to the DAQ system, with a ground reference cable, attached to the user via an elasticated wrist band. These 'active' sensors amplify the EMG signal at the skin surface by 1000 and pass the signal through a high pass filter with a cut-off of approximately $16( \pm 4) \mathrm{Hz}$ and a low pass filter with a cut-off of approximately $460( \pm 15) \mathrm{Hz}$.

An IMU board, sourced from Spark Fun Electronics was also attached to the DAQ device, via screened cable. This consists of an Analog Devices dual axis $\pm 5 \mathrm{~g}$ ADXL320 accelerometer and single axis ADXRS300 $\pm 300 \% \mathrm{~s}$ gyro.

All sensors were powered from an output on the DAQ device, meaning only 1 cable ran from the laptop to the DAQ device.

The system is shown in fig. 1(a), EMG sensors and grounding cable and strap on the right, the insulated IMU at the bottom of the image.

Data are gathered from the USB-1608FS into a Matlab DAQ program, created as a Matlab MEX function, mainly in $\mathrm{C}$ using Microsoft Visual C++ 6 . This interfaced with library code for the DAQ device, allowing the Matlab DAQ program to acquire data while presenting plots of live or past data, together with real-time analysis of the data.

A screenshot of the main control window for the DAQ program is shown in fig. 1(b), and an example EMG recording and its power spectrum can be seen in fig. 2. These data were obtained from EMG recording at the biceps and triceps (solid line and dotted line, respectively in fig. 2) during an elbow flexionextension recording run. The arm was held horizontal with the upper arm held stationary, slightly out to the side of the body, while the lower arm was moved towards and away from the upper sternum by flexing and extending the elbow. The frequency of movement was low to start with, increasing to a higher frequency then reduced again.

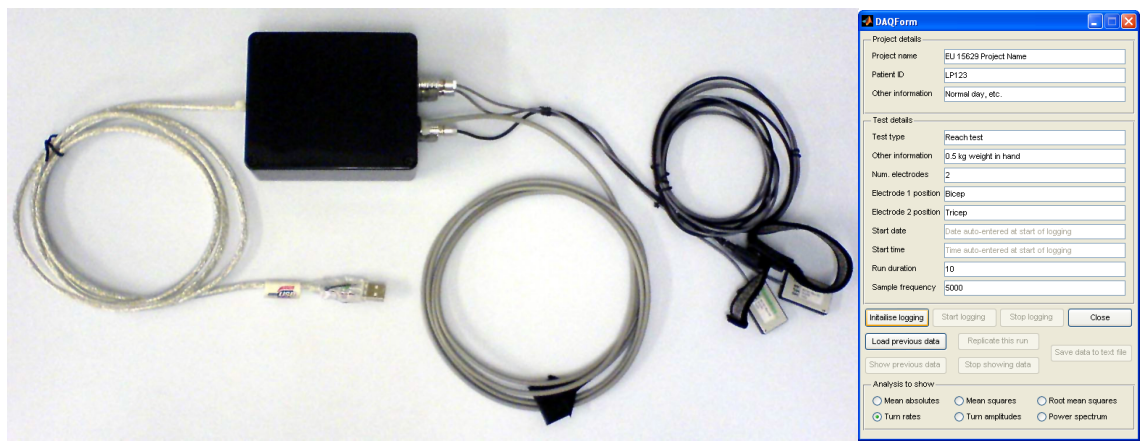

Figure 1: (a) DAQ device, showing USB connection, EMG and IMU sensors, (b) Screenshot of the EMG DAQ control window interface. 


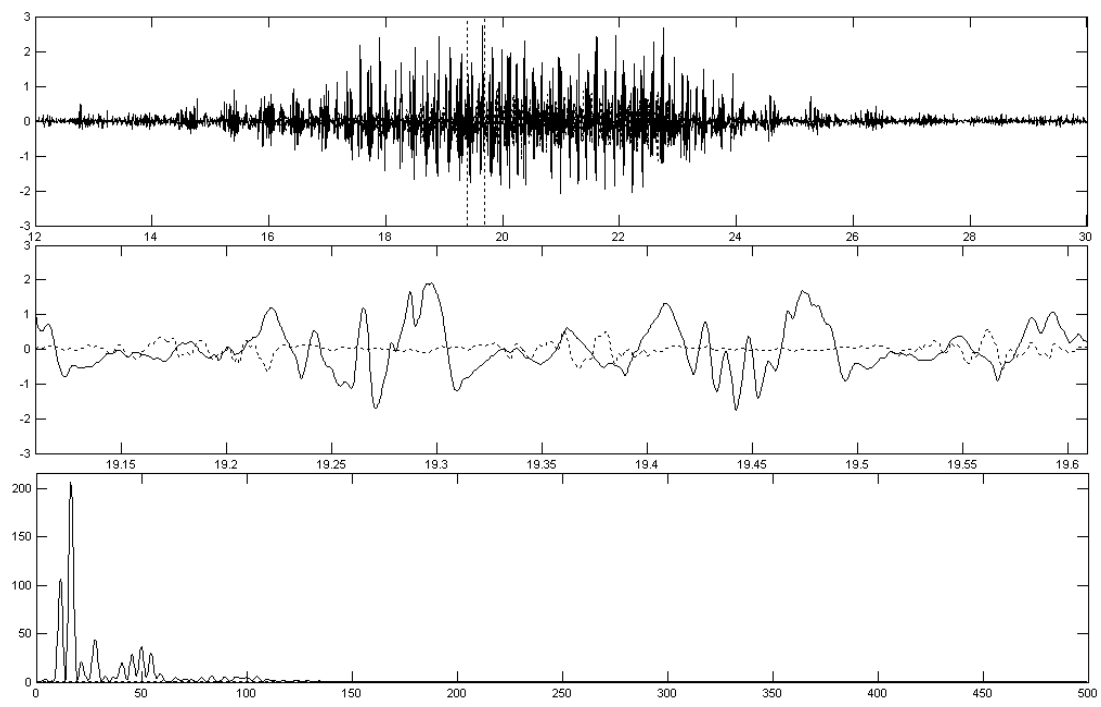

Figure 2: Example EMG, detailed EMG (Time (s) vs. $\mathrm{mV}$ ) and EMG power spectrum data (Frequency (Hz), vs. FFT amplitude ${ }^{2}$ ).

The upper plot of fig. 2 shows a real-time playback of the EMG trace for the section of the run with the flexion-extension movement (12 to $30 \mathrm{~s}$ ).

The second plot shows a detailed view of the EMG pattern, which is otherwise hard to see when the whole run is viewed, as in the upper plot. The plot is zoomed in to $0.5 \mathrm{~s}$ of EMG data, at the point in the run shown in the centre of the dotted box in the upper plot. The central time point of the second plot scrolls through the data in the upper plot, showing the EMG trace in detail while the upper plot gives a better view of the overall EMG signal amplitudes.

Since these data were played back with the "Power spectrum" option selected, the plot in the lower window represents the power spectrum, calculated over a moving window of $0.3 \mathrm{~s}$ of EMG data. The dotted box in the upper plot shows the scope of this moving window, which scrolls through the data, together with the zoomed EMG data, as the run is played back. As can be seen at this point in time there is more activity in the biceps (solid line), and this is reflected in the increased power of the biceps trace on the power spectrum (lower) plot.

Alternative analyses of the data are available during the EMG recording and playback, shown at the bottom of the DAQ program control window in fig. 1(b). These are the mean absolute, mean square, root mean square and power spectrum of the EMG signals over a moving $0.3 \mathrm{~s}$ time window, or values (to the current point in time) of turn rates or turn amplitudes. The turn rates or turn amplitudes are values representing the rates of the 'turns' in the EMG data or amplitudes between 'turn' points. A 'turn' is said to be present in the signal data where there is a local maximum or minimum in the EMG signal (i.e. the signal changes direction) and the "signal amplitude changes by at least $100 \mu \mathrm{V}$ compared to the preceding and subsequent turns", as described by Nandedkar et al [9]. Turns 
were identified using an algorithm based on the description of turns analysis in [9]. These are useful analyses of the EMG interference pattern, as they indicate the force of muscle contraction, and can reveal nerve or muscle problems.

During this run, data were also acquired from the IMU, attached at the wrist so that the gyro was parallel to the lower arm movement, while the first axis of the accelerometer was positioned facing the direction of the (rotational) movement and the second axis was facing along the longitudinal axis of the lower arm, perpendicular to the movement.

The data from this IMU are shown in figs. 3(a) and (b) at the same point in the run as shown in fig 2, the data in fig 3(b) being over a shorter time period. The solid line shows the gyro response, the dotted line indicates the accelerometer response on the first axis (in the direction of the movement) and the dot-dash line the accelerometer response on the second axis (perpendicular to the movement).

It can be seen that the gyro and first accelerometer axis have become saturated (truncated responses at the top and bottom of waves) at this part of the run, during high frequency movements (approximately $5.5 \mathrm{~Hz}$ ), which is at the top of the expected frequency range for tremor this part of the arm. Care should
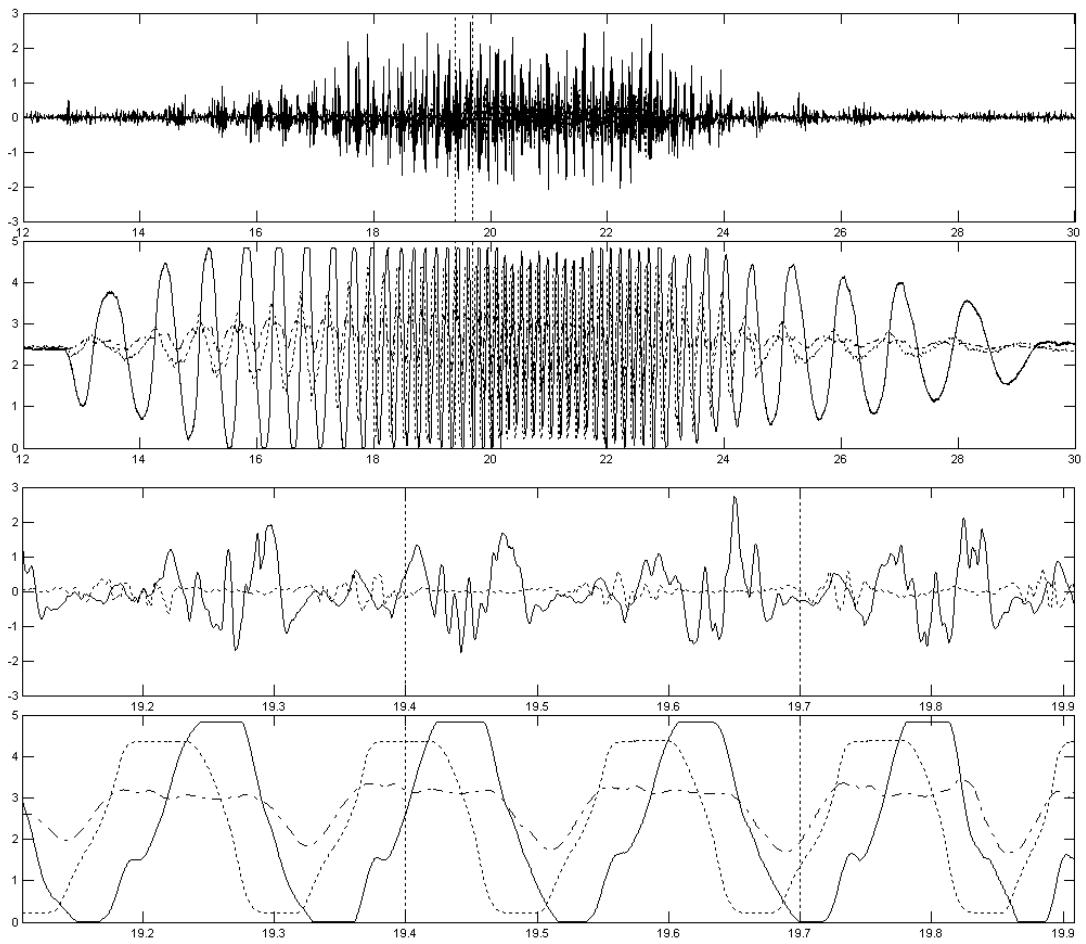

Figure 3: (a) EMG (Time (s) vs. mV) and IMU (Time (s) vs. V), (b) the same data over a shorter time period. 
be taken to choose the most appropriate sensor ranges and axes are to ensure that the readings are always within range, and not artificially truncated. The accelerometer perpendicular to the movement can also be seen to have a fullwave rectified appearance, as the acceleration acting on that axis is always in the same direction, no whether the elbow is flexing or extending.

\subsection{SimMechanics model}

A model was created in SimMechanics to simulate the dynamics of the upper limb, including shoulder movements, shown in fig. 4. SimMechanics is a Matlab $^{\circledR}$ toolbox that enables rigid body systems to be built, with rotations between bodies, in a simple block-based interface. The model incorporates the major rotations of the upper limb as two rotations between the main torso and shoulder, three rotations at the shoulder (glenohumeral joint), one rotation at the elbow, a rotation in the lower arm and two rotations at the wrist.

The rotation about the longitudinal axis of the upper and lower arm segments are modelled as a rotation partway along each segment, as rotations experienced at the skin, where movement sensors will be located, experience little rotation at the proximal end of the segment, but far more or the rotation of the solid bone structure beneath is transmitted to the skin towards the distal ends.

Bodies with realistic masses and centres of mass (derived from data in [10]) together with suitable inertias were included between the joint rotations, which are themselves included in subsystems in the model.

The elbow sub-system (shown highlighted in the model) is shown expanded in the box within fig. 4 . This example shows a simple sin wave and its single and double differentials feeding into the elbow joint to give angles, angular rates and angular accelerations over time. Running this model makes the lower arm reciprocate through regular angular movements (see fig. 5) and feeds the angles rotated through and torque about the elbow joint to the to the Matlab workspace, enabling manipulation after completion of the run.

Converting measured elbow angles into time-variant elbow angle, angular rate and angular acceleration data and feeding these into the model enables assessment of the forces involved in both the intentional and tremulous parts of movements with intention tremor. If it were assumed that the tremor forces remained the same regardless of devices used to impede the forces, it would enable virtual trials of equipment to reduce the tremor and so help in the design of optimal equipment and control strategies. This would provide a first estimate of the devices best suited to reducing tremor at the elbow during movements. However, it is likely that the systems involved in the nervous control of skeletal muscles would react to inertial and other resistances provided by tremor reduction devices in ways that would make this assumption invalid. In order to obtain a more meaningful result, an approximation of the nervous control system and muscle dynamics need to be incorporated into the model, incorporating the ability to model 'normal' movements and those affected by intention tremor.

Once developed on offline data, this model can be adapted to enable real time control of tremor by taking in orientation and movement data from the Xsens IMUs and outputting signals to control devices to reduce the tremor. 


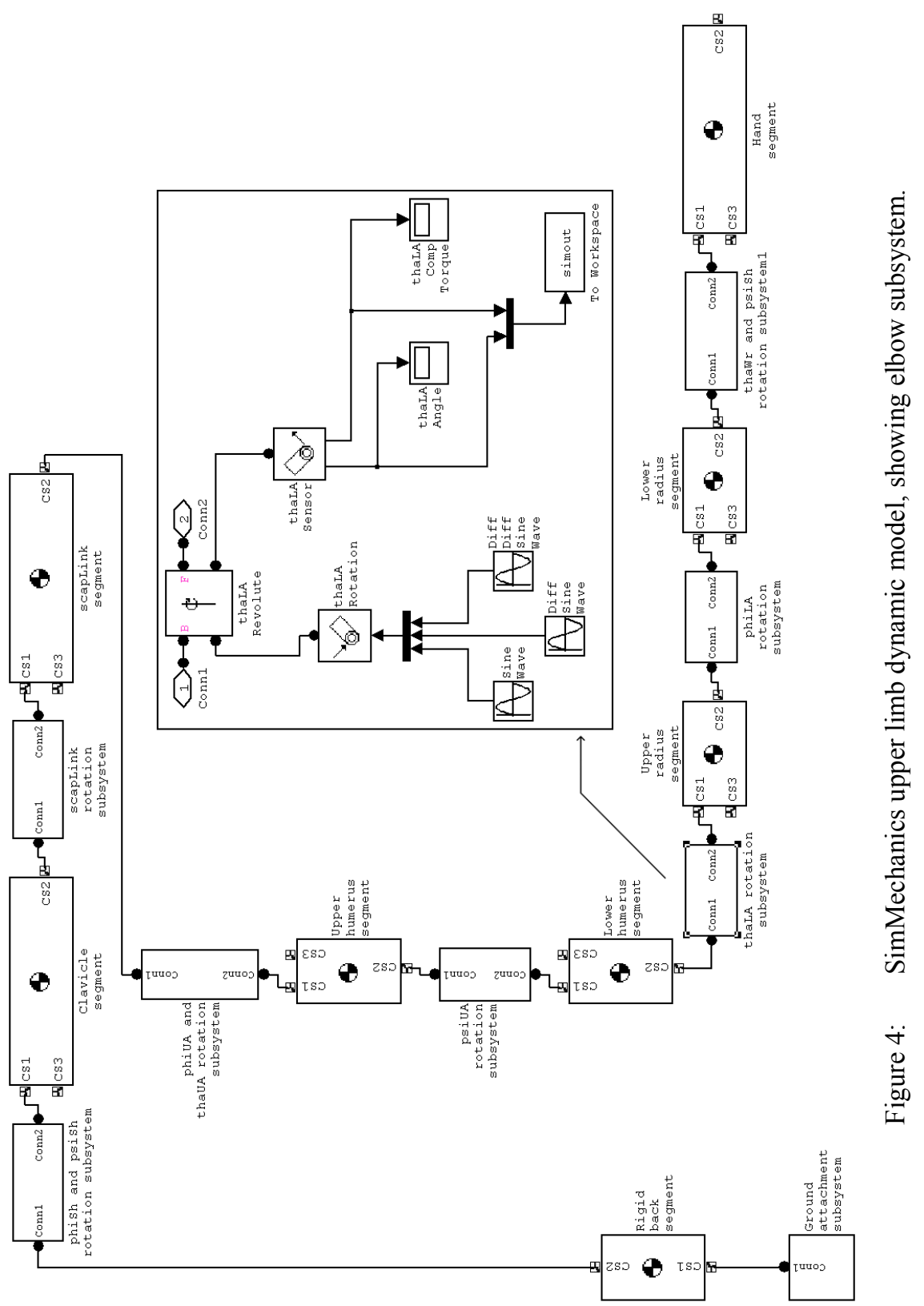




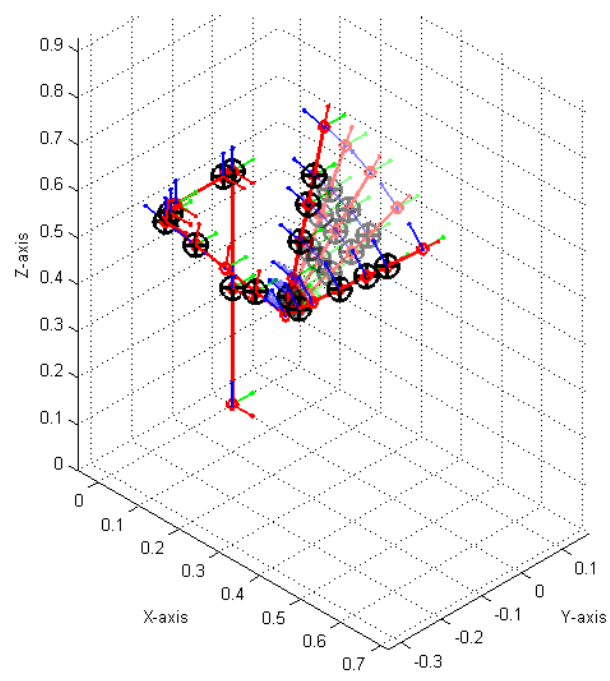

Figure 5: SimMechanics machine of the upper limb moving the lower arm through a set of angle data while all else is stationary.

The SimMechanics environment has the added advantage that it is easier to use frequency domain techniques, such as composite filters, to combine measurement device readings, as described by Hyde et al [7]. In this way, the most appropriate readings from the accelerometers, gyros and magnetometers contribute to the estimate of the body movement in the frequency range to which they are best suited. Other tools that are available in Matlab and its toolboxes should assist with development of accurate position and movement estimation.

\section{Conclusion}

A project to measure and control intention tremor in the upper limbs of individuals with MS, and its future directions, is described. Dynamic modelling of the upper limb plays a central part to understanding the movements and forces involved in tremulous arm movements and creating systems to reduce this tremor, enhancing the daily lives of those affected by MS intention tremor.

\section{References}

[1] Calabresi, P.A., Diagnosis and management of multiple sclerosis. American Family Physician, 70(10), pp. 1935-1944, Nov 2004.

[2] Prat, E. \& Martin, R., The immunopathogenesis of multiple sclerosis. Journal of Rehabilitation Research and Development, 39(2), pp. 187-199, 2002.

[3] Alusi, S.H., Worthington, J., Glickman, S. \& Bain, P.G., A study of tremor in multiple sclerosis. Brain, 124(Pt 4), pp. 720-30, Apr 2001. 
[4] Memberg, W. D., Crago, P. E. and Keith, M. W., Restoration of elbow extension via functional electrical stimulation in individuals with tetraplegia. Journal of Rehabilitation Research and Development, 40, pp. 477-486, 2003.

[5] Giansanti, D., Macellari, V., Maccioni, G. and Cappozzo, A., Is it feasible to reconstruct body segment 3-D position and orientation using accelerometric data? IEEE Transactions on Biomedical Engineering, 50, pp. 476-483, 2003.

[6] Giansanti, D., Macellari, V., Maccioni, G. and Cappozzo, A., The development and test of a device for the reconstruction of 3-D position and orientation by means of a kinematic sensor assembly with rate gyroscopes and accelerometers. IEEE Transactions on Biomedical Engineering, 52, pp. 1271-1277, 2005.

[7] Hyde, R.A., Ketteringham, L., Neild, S.A. and Jones, R.J.S., Estimation of Upper-Limb Orientation Based on Accelerometer and Gyroscope Measurements. IEEE Transactions on Biomedical Engineering, awaiting publication in 2007.

[8] Dejnabadi, H., Jolles, B.M. and Aminian, K., A new approach to accurate measurement of uniaxial joint angles based on a combination of accelerometers and gyroscopes. IEEE Transactions on Biomedical Engineering, 52, pp. 1478-1484, 2005.

[9] Nandedkar, S.D., Sanders, D.B. and Stalberg, E.V., Automatic analysis of the electromyographic interference pattern. Part I: Development of quantitative features. Muscle Nerve, 9(5), pp. 431-439, Jun 1986.

[10] Dempster, W.T., Space requirements of the seated operator. WADC Technical Report, Wright-Patterson Air Force Base, Ohio, 1955. 